\title{
CORTICAL AND SUBCORTICAL BRAIN ALTERATIONS INPATIENTSWITH SYSTEMIC SCLEROSIS: A LONGITUDINAL MAGNETIC RESONANCE IMAGING (MRI) STUDY
}

Danilo Rodrigues Pereira ${ }^{1}$, Mariana Freschi Bombini ${ }^{1}$, Renan Bazuco Frittoli ${ }^{1}$, Ana Carolina Londe ${ }^{1}$, Tiago Amaral ${ }^{1}$, Sergio Dertkigil ${ }^{1}$, Fernando Cendes $^{1}$, Leticia Rittner ${ }^{1}$, Simone Appenzeller ${ }^{1, *}$

1.Universidade Estadual de Campinas, Campinas (SP), Brazil.

*Corresponding author: appenzellersimone@gmail.com

\section{BACKGROUND}

Systemic sclerosis (SSc) is classified as autoimmune connective tissue disease. However, central nervous system (CNS) involvements are often observed. Magnetic resonance imaging (MRI) is the important technique for studying structural brain changes. The aim of the present study was to analyze differing cortical and subcortical structural alterations in SSc patients.

\section{METHODS}

Twenty-nine patients with SSc were recruited (mean age 52.9 years; SD \pm 13.5). Three-dimensional (3D) T1-weighted MRI images were acquired using 3T Philips MRI scanner. After 48 months, MRI acquisitions were repeated in all $39 \mathrm{SSc}$ patients. All subjects had normal MRI at visual inspection. Cortical and subcortical volumes were calculated with FreeSurfer software. The volumetric structures measures analyzed were the putamen, caudate nucleus, globus pallidus, nucleus accumbens, thalamus, amygdala, hippocampus, and the ventricular system. All SSc patients were further assessed for clinical and laboratory SSc manifestations, Beck's depression (BDI), Beck's anxiety inventories (BAI), Montreal cognitive assessment (MoCA) and current drug exposures. Demographical data and clinical characteristics of the patients were analyzed using IBM SPSS statistics software (version 25.0) and parametric and nonparametric statistics were used as appropriate.

\section{RESULTS}

We observed significant reduction in cortical and subcortical brain structures volumes in SSc patients when compared to follow-up patients: Left lateral ventricle ( $p=0.001$ ); hippocampi (left: $p=0.008$, right: $p<0.001$ ), left hippocampus associated with anxiety symptoms $(p=0.019)$ and right associated with MoCA scores $(p=0.047)$; amygdala (left and right: $p<0.001)$, right amygdala associated with headache ( $p=0.029$ ); thalamus (left and right: $p<0.001$ ), left thalamus associated with use of prednisone $(p=0.036)$; caudate (left and right: $p<0.001$ ); left putamen $(p<0.001)$ associated with use of prednisone $(p=0.024)$; pallidum (left and right: $p<0.001)$; accumbens (left and right: $p<0.001)$, right accumbens associated with use of antidepressant $(p=0.046)$ and depression symptoms ( $p=0.025)$; cerebellum ( $p<0.001$ ) associated with depression symptoms $(p=0.006)$, use of methotrexate ( $p=0.046$ ) and depression symptoms ( $p=0.015)$; cerebrospinal fluid (CSF) $(p<0.001)$; corpus callosum (CC) mid anterior $(p<0.014)$ associated with use of prednisone ( $p=0.013)$; CC mid posterior $(p<0.047)$ associated with use of prednisone $(p=0.001)$ and presence of digital ulcers $(p=0.033)$.

\section{CONCLUSION}

The results of this study demonstrated the cortical and subcortical structures volumes were significantly reduced in SSc patients when compared to follow-up patients. Volumetric measures reductions were associated with the presence of digital ulcers, anxiety symptoms, depression symptoms, MoCA scores, headache and use of prednisone, antidepressant and methotrexate during the treatment.

\section{KEYWORDS}

Systemic sclerosis, Magnetic resonance imaging, Cortical, Subcortical. 